tural results. It's last section discusses archiving of final results. Chapter 16 gives an excellent introduction to the preparation and use of the CIF and Chapter 17 lists the various crystallographic databases with primary emphasis on the Cambridge Structural Database. Chapter 18 deals with twinning, anomalous dispersion and X-ray sources.

Three appendices present mathematics and formulae, a crystallographic dictionary, and answers to exercises.

This textbook should definitely be considered for use in introductory courses in $\mathrm{X}$-ray structure determination as it provides a good framework for course organization. The concise treatment of the material (all of small-molecule X-ray crystallography in 265 pages) will work well when supplemented with lectures and additional class discussions.

\section{Gary Newton}

Department of Chemistry

University of Georgia

Athens

GA 30602

USA

Perovskites modern and ancient. By Roger H. Mitchell. Thunder Bay, Ontario: Almaz Press, 2002. Price USD 70.00. ISBN 0-9689411-0-9

Perovskites, whose general formula is given by $A B X_{3}$, where $A$ and $B$ are cations and $X$ is an anion, form a very important class of inorganic crystals whose physical properties are extensively used in many technological applications. These generally arise from the large range of pseudosymmetrically related crystal structures. The basic, so-called aristotype structure, consists of an infinite array of corner-linked anion octahedra, with the $A$ cations in the spaces between the octahedra and a $B$ cation at the centre of each octahedron. This structure can then be altered by allowing the cations to move away from their central positions, either together, in which case one has polar structures, or antiparallel. In addition, the octahedra can tilt about different directions, giving rise to multiple unit cells, and they can be distorted. For the crystallographer they are a delight as the vast range of structures can furnish a lifetime of study.

What is surprising is that, apart from one text published by F. S. Galasso many years ago, no-one until now has put together a complete account in English on this intriguing family of structures. There was a good treatment written in Russian by K. S. Aleksandrov and B. V. Beznosikov in 1997, but as far as I know it was not translated into English. This new book by Roger Mitchell addresses this gap in a such a splendid manner. The book is dedicated to my old boss, Dr Helen D. Megaw, who pioneered so much of our knowledge of the perovskite structure: personally, I feel that the author could not have chosen a better person. This is the book that I wish I had written!

So what about the book itself? The first thing that strikes one is the quality of the production. This is a book in which colour has been used to considerable good effect almost a work of art. It is, put simply, a beautiful book, and the author has obviously worked extremely hard to present his subject in as clear and as eye-catching manner as possible. But I should emphasize that it is not just a matter of presentation that makes this book so worthwhile. Roger Mitchell has in fact produced such a thorough, well researched description of all aspects of the perovskite structure that I believe it will become a classic in this field. It is stuffed full of factual material, lavishly illustrated and informative. However, having waxed lyrical about this book, I hope the author will forgive me one gripe. There is no subject index, the author preferring instead to point to an extended table of contents. This is a pity as it makes it hard to look up particular items. For instance, I had difficulty in trying to locate a discussion of incommensurability in these structures.

The book consists of ten chapters in a nice logical sequence. It begins with a description of the ideal structure and related compounds, and then proceeds to describe how this structure can be altered, using geometric and structural principles. The author makes much use of the concept of octahedral tilting to explain the many structural types as well as the effect of cation displacements. A really nice feature is the inclusion of many powder diffraction diagrams to illustrate how one can distinguish between the types, including close-ups of particular diffraction peaks to illustrate splittings distinguishing symmetries. Chapter 3 considers what happens on mixing perovskite compounds to form solid solutions, and Chapter 4 discusses cation ordering, both at the $A$ and $B$ sites. Following this, non-stoichiometric perovskites, i.e. those with vacancies and defects, are described.

Subsequent chapters cover related materials, such as hexagonal perovskites, layered perovskites, including those familiar to scientists working on high- $T_{c}$ superconducting phases. Finally the effect of high pressure on silicate perovskites and naturally occurring perovskites are dealt with. These last two chapters will be of prime interest to earth scientists, since perovskites such as $\mathrm{MgSiO}_{3}$ form large parts of the earth's mantle.

Summarizing then, this book should be read by everyone interested in perovskites, or for that matter, inorganic structures in general. It is bang up-to-date, and as such it is a vital reference in the field. I am delighted to have a copy and it will occupy pride of place on my bookshelf for a very long time.

\section{A. M. Glazer}

Department of Physics

Clarendon Laboratory

Parks Road

Oxford OX1 3PU

UK

\section{books received}

The following books have been received by the Editor. Brief and generally uncritical notices are given of works of marginal crystallographic interest; occasionally, a book of fundamental interest is included under this heading because of difficulty in finding a suitable reviewer without great delay.

\section{Fundamentals of solid-state phase transitions, ferromagnetism and ferroelectricity. By Yuri Mnyukh.} Bloomington, IN: 1st Books Library, 2002. Price USD 23.95 (hardback), USD 18.95 (paperback), USD 16.95 (electronic book 8350k). ISBN 0-75960-219-0. Theory and experiments on solid-state phase transitions, especially relating to ferromagnetism and ferroelectricity, have a very long history. They are documented and discussed in substantial books and a voluminous literature. This review of the subject, following a Critical Survey, has chapters on the Molecular mechanism of solid-state phase transitions, 'Lambdaanomalies' and other apparent anomalies, and Fundamentals of ferromagnetism and ferroelectricity. Throughout the text there are conventional and alternative descriptions offered of these phenomena as well as six appendices describing earlier debates. 